\title{
Pair congruence and interpair interference in paired-associate learning
}

\author{
MAUREEN CLEMENTS and TANNIS Y. ARBUCKLE. \\ Department of Psychology. Sir George Williams Unirersity, \\ Montreal 25, Canada
}

Pair congruence (C) is the number of times any given pair is constructed when Ss freely pair two sets of items. Interpair interference (I) is estimated from degree of $C$ between any pair's members and other list items. One hundred Ss learned one of five 6-pair lists, each list representing a different combination of $C$ and $I$, and then reported mnemonics. Increasing $C$ facilitated learning, while varying I had no systematic effect. Mnemonics were reported more frequently and were less idiosyncratic when $C$ was high, suggesting that $C$ values reflect degree of availability of mediators.

Pair congruence (C) for any A-B pair may be defined as the number of Ss out of a sample of size $N$ who formed that pair when given the task of pairing, on whatever basis they deemed appropriate, P A items with P B items to form P A-B pairs. Richardson (1968) found that, given constant sets of $A$ and $B$ members, a list of A-B items for which a mediator, inferred from association norms, was presumed to be available for chaining $A$ and $B$, was learned more slowly than was a list of $A-B_{r}$ items in which the items had been re-paired to provide the highest possible $C$ value for each pair. Richardson attributed his results to the high pair $C$ values, which he assumed reflected the existence of previously learned direct associations between $\mathrm{A}$ and $\mathrm{B}$, but he noted that the results might also have been due to a concomitant reduction in interpair interference in the A-B $B_{r}$ list. Interpair interference (I) for any pair may be defined as the degree to which its $A$ and $B$ members are associated through prior learning with the $B$ and A members, respectively, of other pairs on the list.

In the present experiment we manipulated $C$ and $I$ across lists so as to determine the effects of each variable on paired-associate (PA) learning. The $\bar{C}$ value for any list was the mean of the $C$ values of the $L$ pairs, where $L=$ list length. The $\bar{I}$ value for any list was the mean of the $C$ values of two sets of $\mathrm{L}$ pairings, the first set being the pairings having maximum $\mathrm{C}$ value between each $A$ member and any $B$ member of any other pair on the list, and the second being the corresponding set of $B$ to A pairings.

\section{METHOD}

The Ss were 100 male and female undergraduates who were paid for their services.

The materials were five lists of six PA items whose A and B members came from the low and high extremes, respectively, of Noble's (1952) $m$ scale. Congruence values for these pairs were available from an unpublished study conducted under the second author's direction using different Ss in which $N=62$ and $P=16$. Items were photographed on slides and presented to Ss, tested individually. by a Carousel projector at a $3 \mathrm{sec}$ rate.

Three levels of $\mathrm{C}$ and $\mathrm{I}$ were used: High (h), Moderate (m), and Low (1). Complete testing of all nine combinations would require nine lists; however, because of the limited number of pairs scaled for congruency, only five were actually used: $C_{h} I_{l}$ $(\overline{\mathrm{C}}=18.0, \overline{\mathrm{I}}=3.8) ; C_{\mathrm{m}} \mathrm{I}_{1}(\overline{\mathrm{C}}=6.3, \overline{\mathrm{I}}=5.8) ; \mathrm{C}_{\mathrm{m}} \mathrm{I}_{\mathrm{h}}(\overline{\mathrm{C}}=6.0$ $\overline{\mathrm{I}}=14.3) ; \mathrm{C}_{1} \mathrm{I}_{\mathrm{m}}(\overline{\mathrm{C}}=.8, \overline{\mathrm{I}}=7.1) ;$ and $\mathrm{C}_{1} \mathrm{I}_{\mathrm{h}}(\overline{\mathrm{C}}=.8, \overline{\mathrm{I}}=18.0)^{2}$ Twenty Ss were randomly assigned to each list condition. Five between-group comparisons were planned: (a) $C_{h} I_{1}$ vs $C_{1} I_{h}$-both $C$ and I varied maximally: (b) $C_{m} I_{1}$ vs $C_{1} I_{m}$ - both C and I varied minimally; (c) $C_{h} I_{1}$ vs $C_{m} I_{1}-C$ varied minimally, I constant; (d) $C_{m} I_{1}$ vs $C_{m} I_{h} C$ constant. I varied maximally; and (e) $C_{1} I_{m}$ vs $C_{1} I_{h}-C$ constant, I varicd minimally.

The recall method of PA learning was used with training continuing to a criterion of three successive trials. Five different orders of presentation and testing of items were used with approximately equal frequency across trials for each $S$. After reaching criterion, $S s$ were asked to report all mmenonics used.

\section{RESULTS}

As Columns 1 and 2 of Table 1 show, both trials to criterion (inclusive of the three criterion trials), and errors to criterion tended to increase as $C$ decreased. The effect of changing $I$, with $\mathrm{C}$ held constant, tended to be small and no consistent trend was observed. Of the five planned comparisons, the only one to attain significance on the Mann-Whitney $U$ test was comparison (a), with List $C_{h} I_{1}$ requiring significantly fewer trials to learn $(p<.02)$ and producing significantly fewer errors $(p<.05)$ than did List $C_{1} I_{h}$. A post hoc analysis, using the method suggested by Dunn (1964), showed that performance on List $C_{h} l_{1}$ was significantly superior to performance on all other lists combined on both the trials measure $(z=2.7 ; p<.01)$ and the errors measure $(z=2.0$ $\mathrm{p}<.05$ ).

The total number of mnemonics $(n)$ reported for each list are shown in Column 3 of Table 1 . with List $C_{h} I_{1}$ clearly eliciting more reported mnemonics than any other list. The mnemonics reported by $S s$ were rated by two independent judges as to similarity of concepts. For example, for one A-B pair the mnemonics reported included "not," "no," "negative" and "negligent." The first three were judged as representing the same concept and the fourth a different one. The classifications of the two judges across conditions were similar and hence were combined. The mean number of dissimilar mnemonics, as rated by the two judges, was found

Table 1

Trials, Errors and Mnemonics for Five Conditions of $\mathrm{C}$ and I

\begin{tabular}{lcccc}
\hline List & $\bar{X}$ Trials & $\bar{X}$ Errors & n Mnem. & p Diff. Mnem. \\
\hline$C_{h} I_{1}$ & 6.25 & 9.50 & 93 & .28 \\
$C_{m} I_{1}$ & 7.80 & 11.60 & 60 & .47 \\
$C_{m} I_{h}$ & 7.95 & 11.20 & 69 & .50 \\
$C_{l} I_{m}$ & 9.50 & 16.45 & 54 & .57 \\
$C_{1} I_{h}$ & 8.45 & 13.85 & 69 & .57 \\
\hline
\end{tabular}


for each list and was expressed as a proportion of the total $n$ for that list. This proportion could vary from a high of 1.0 , where each item elicited a different concept from each $S$, to a low of $L / n$, where each item elicited the same concept from all Ss. As Column 4 of Table 1 shows the propertions decreased as $\mathrm{C}$ increased. When the five planned comparisons were made between groups, using the $\mathrm{z}$ test for differences between independent proportions, comparisons (a) and (b) were significant, with List $C_{h} I_{1}$ eliciting proportionately fewer dissimilar mnemonics than did either List $C_{1} I_{h}$ or List $C_{m} I_{1}$.

\section{DISCUSSION}

The decrease in both trials and errors to criterion as $\mathrm{C}$ increased agreed with Richardson's finding that $C$ is an important determiner of PA learning. That this effect was statistically significant only when $\mathrm{C}$ and $\mathrm{I}$ were both varied maximally was probably because, with the short list length, all Ss reached criterion rapidly and therefore the size of differences among groups was restricted. Varying I with $C$ held constant had no consistent effect on trials and errors. It is possible that the failure to demonstrate slower learning with increasing 1 was also an artifact either of list length, or of the more restricted range of $I$ as compared to $C$, though it must be noted that in three out of four of the relevant comparisons the trend was in the opposite direction.

Richardson has suggested that the effect of $\mathrm{C}$ and $\mathrm{I}$ on learning depends not on their absolute values, but rather only on which is the greater, hence one would not always expect variation in C or I to affect learning. However, this suggestion, applied to the present experiment, would predict significant differences on Comparisons (a), (b), and (d), in each of which $\mathrm{C}-\mathrm{I}$ was positive for the one list and negative for the other, and no differences on Comparisons (c) and (e), in each of which $\mathrm{C}-\mathrm{I}$ had the same sign for both lists. On this basis we would have predicted correctly in three cases (a, c, and e) and incorrectly in the other two, a not overly impressive record. Pending further investigation it seems most reasonable to conclude that PA acquisition is affected by $C$, while the effect of $I$ is questionable.

The finding that List $C_{h} I_{1}$ elicited more mnemonics, yet proportionately fewer different mnemonics than the other lists, suggested that high $C$ pairs may be learned faster because an appropriate mediator is readily available to most Ss learning the list. Richardson implied that high $\mathrm{C}$ pairs were quickly learned because a strong direct association pre-existed between the $A$ and $B$ members; we would argue that the $C$ value of a pair measures the degree of availability of mediators. On this basis we would speculate that Richardson's A-B lists were learned faster than his A-(m)-B lists simply because a mediator was more readily available for high $\mathrm{C}$ pairings than for pairings based on association norms.

\section{REFERENCES}

DUNN, C. J. Multiple comparisons using ranks. Technometrics, 1964, 6, 241-252.

NOBLE, C. E. Analysis of meaning. Psychological Review, 1952, 59, $421-430$.

RICHARDSON, J. Implicit verbal chaining as the basis of transfer in paired-associate learning. Journal of Experimental Psychology, 1968, 75, 109-119.

\section{NOTES}

1. This research was supported by Grant APA 209 from the National Research Council of Canada. This paper is based on the B. A. (Hons.) thesis done by the first author under the direction of the second author. The authors wish to thank Gerrie Alborg for collecting the congruency data.

2. The order of magnitude of the $C$ and I values of individual pairs in each list was that specified by the $\overline{\mathrm{C}}$ and $\overline{\mathrm{I}}$ values of the list, except for one pair in List $C_{m} I_{1}$ and one in List $C_{m} I_{h}$. These exceptions were necessitated by the difficulty in constructing lists where all $6 \mathrm{C}$ pairs and all 60 possible I pairs conformed to the list specifications. 